\title{
Lehrkräfte in der Erwachsenen- und Weiterbildung - Zusammenhänge zwischen Vorbildung und Erfahrung mit dem Wissen über Lehr-Lernmethoden und -konzepte
}

\author{
Christian Marx • Annika Goeze $・$ Augustin Kelava $・$ Josef Schrader
}

Eingegangen: 2. März 2018 / Angenommen: 24. Mai 2018 / Online publiziert: 8. Juni 2018

(C) Der/die Autor(en) 2018

Zusammenfassung Das Wissen über Lehr-Lernmethoden und -konzepte gilt als ein wichtiger Aspekt des pädagogisch-psychologischen Wissens von Lehrkräften in der Erwachsenen- und Weiterbildung (EB/WB) für die Gestaltung von Lehr-Lernsituationen. Der Beitrag untersucht, ob und inwiefern dieses Wissen mit der Vorbildung und der Erfahrung von $N=212$ Lehrkräften aus der EB/WB zusammenhängt. Die Ergebnisse weisen darauf hin, dass der Umfang an Fort- und Weiterbildungen zum Thema Lehren und Lernen/Methodik/Didaktik in einem positiven Zusammenhang mit dem erfassten Wissen steht und Lehrkräfte, die in der beruflich-betrieblichen Weiterbildung tätig sind, im Vergleich betrachtet über relativ viel Wissen verfügen. Keine (direkten) Zusammenhänge zu diesem Wissen zeigten sich entgegen der Annahmen zur Lehrerfahrung sowie einem absolvierten erziehungs- und bildungswissenschaftlichen Studium. Die Befunde, insbesondere letzterer, werden hinsichtlich ihrer Belastbarkeit und Reichweite kritisch diskutiert und an den aktuellen Diskurs der EB/WB angeschlossen.

Publisher's Note Springer Nature remains neutral with regard to jurisdictional claims in published maps and institutional affiliations.

C. Marx $(\bowtie) \cdot$ Dr. A. Goeze $\cdot$ Prof. Dr. J. Schrader

Leibniz-Zentrum für Lebenslanges Lernen e. V., Deutsches Institut für Erwachsenenbildung,

Heinemannstraße 12-14, 53175 Bonn, Deutschland

E-Mail: marx@die-bonn.de

Dr. A. Goeze

E-Mail: goeze@die-bonn.de

Prof. Dr. J. Schrader

E-Mail: schrader@die-bonn.de

Prof. Dr. A. Kelava

Methodenzentrum der Wirtschafts- und Sozialwissenschaftlichen Fakultät, Eberhard Karls

Universität Tübingen, Wilhelmstr. 31, 72070 Tübingen, Deutschland

E-Mail: augustin.kelava@uni-tuebingen.de 
Schlüsselwörter Pädagogisch-psychologisches Wissen · Lehr-Lernmethoden und -konzepte $\cdot$ Lehrkräfte $\cdot$ Trainer $\cdot$ Erwachsenenbildung/Weiterbildung

\title{
Teachers and trainers in adult and further education: relations between their educational background and teaching experience with their knowledge about teaching methods
}

\begin{abstract}
Teachers' knowledge about teaching methods is an important facet of adult education teachers' pedagogical/psychological knowledge (PPK). The authors examine whether and how this knowledge is correlated with the educational background and the teaching experience of $N=212$ adult education teachers. The results point to a positive correlation between the amount of further training concerning the topic teaching and learning and the knowledge about teaching methods. Moreover, teachers with a focal point on vocational training showed a relatively high amount of knowledge about teaching methods. Contrary to the assumptions, there was no (direct) correlation between teachers' teaching experience and teachers' knowledge as well as between teachers' pedagogical education and teachers' knowledge. Finally, results are discussed with regard to their reliability and reach as well as to their contribution to the present discourse in the field of adult education.
\end{abstract}

Keywords Pedagogical/psychological knowledge $\cdot$ Knowledge about teaching methods · Teachers · Trainers · Adult education

\section{Einleitung: Zur Entwicklung professioneller Kompetenz von Lehrkräften in der Erwachsenen- und Weiterbildung als Forschungsdesiderat}

Befragungsdaten aus der Erwachsenen- und Weiterbildung (EB/WB) (z. B. Hartz 2011), Expertisen für die Politik (Guerriero 2017; Kraft et al. 2009) und auch empirische Studien aus dem Schulbereich (Rivkin et al. 2005) verweisen auf den großen Einfluss des pädagogischen Handelns von Lehrkräften auf den Lernerfolg der Lernenden. Demgegenüber wird den institutionellen Rahmenbedingungen bildungsbereichsunabhängig zumeist deutlich weniger Einfluss zugeschrieben. Folglich wuchs in der EB/WB in den vergangenen Jahren das Interesse an der Erforschung einzelner Bestandteile der wie folgt angenommenen Wirkungskette (Terhart 2012): (1) Ausund Weiterbildung von Lehrkräften führen zu (2) professioneller Kompetenz und darüber vermittelt zu (3) hoher Qualität bei Lehr-Lernprozessen, die (4) den Lernerfolg bei Teilnehmenden befördert.

An der konkreten Aus- bzw. Weiterbildung von Lehrkräften in der EB/WB setzen zahlreiche Initiativen für die Praxis an (z.B. Avramovska et al. 2015; Lencer und Strauch 2016; Schläfli und Sgier 2008; Schöb et al. 2016; Steiner 2010); verschiedene Bereiche der professionellen Kompetenz von Lehrkräften sind in den Fokus empirischer Forschung gerückt (Hof 2010; Lattke und Jütte 2014; Pachner 2013; Rohs et al. 2017; Stanik 2016; Vinepac-Project 2008) ebenso konkrete LehrLernsituationen (Herrle et al. 2014) sowie die Kompetenzen der Teilnehmenden 
(Rosenbladt und Lehmann 2013). Bislang gibt es allerdings nur wenig Forschung in der EB/WB, die einzelne Glieder in ihrer Interdependenz untersucht (s. aber z. B. Goeze et al. 2013).

Betrachtet man die ersten beiden Glieder der Kette, so kann man - anders als im Schulkontext, aber ähnlich dem Hochschulkontext - relativ wenig systematische und institutionalisierte Einflussnahme auf die Entwicklung der professionellen Kompetenz von Lehrkräften in der EB/WB konstatieren. Weil der Ausbildungsund Berufszugang sowie die Berufsausübung hier nur in geringem Maße staatlich, tarifvertraglich oder berufsständisch reglementiert und damit seit jeher „offen“ für Lehrkräfte mit unterschiedlichem Ausbildungshintergrund sind, bleibt die Entwicklung von professioneller Kompetenz eine (größtenteils) individuelle ,berufs-“biografische Entscheidung jeder einzelnen Lehrkraft und ist daher vergleichsweise schwer $\mathrm{zu}$ untersuchen. Umso mehr ist es von Interesse für Forschung und Praxis, welche Vorbildungen und Erfahrungen mit der professionellen Kompetenz(entwicklung) der Lehrkräfte (,on the job“) in Zusammenhang stehen könnten.

Das Wissen um die berufsbiografische, berufliche und soziale Situation von EB/WB-Lehrkräften konnte in den vergangenen Jahren durch mehrere Studien zum Personal in der Weiterbildung deutlich verbessert werden (Autorengruppe Bildungsberichterstattung 2016; Autorengruppe wb-personalmonitor 2016; Dobischat et al. 2018). Dabei wurden grundlegende Merkmale sowie deren typische Ausprägungen identifiziert, von denen angenommen werden darf, dass sie die Entwicklung der professionellen Kompetenz von Lehrkräften und in der Folge die Gestaltung von LehrLernsituationen begünstigen könnten (Schrader und Goeze in Vorbereitung).

Neben den Überzeugungen von Lehrkräften (z. B. Collins und Pratt 2011), die relativ zeitstabil sind (z.B. zusammenfassend Skott 2015), wird ein Bereich der professionellen Kompetenz von Lehrkräften derzeit innerhalb der EB/WB verstärkt thematisiert: Das vergleichsweise gut zu entwickelnde professionelle Wissen von Lehrkräften (Marx et al. 2017; Rohs et al. 2017). Welche ausbildungs- und tätigkeitsbezogenen Merkmale für die Lehrtätigkeit einschlägige Lern- und damit Kompetenzentwicklungsgelegenheiten darstellen können (z. B. ein erziehungs- und bildungswissenschaftliches Studium, Fort- und Weiterbildungen, Tätigkeitsumfang und Lehrerfahrung sowie die Tätigkeitsschwerpunkte) und wie diese mit dem professionellen Wissen von EB/WB-Lehrkräften zusammenhängen, wurde bislang noch nicht untersucht. Ein Grund hierfür kann sein, dass es an standardisierten Instrumenten insgesamt bislang mangelt(e), siehe aber das Teaching Perspectives Inventory (Collins und Pratt, 2011) und das Validpack-Instrument (Vinepac-Project 2008). Insbesondere Instrumente zur Erfassung (einzelner Facetten) des professionellen Wissens sind erst seit jüngster Zeit in der Entwicklung und Validierung (Marx et al. 2017; Rohs et al. 2017).

Ein im Rahmen der genannten Lern- und Kompetenzentwicklungsgelegenheiten viel thematisierter Bereich des professionellen Wissens von Lehrenden ist das Wissen über Lehr-Lernmethoden und-konzepte (Schrader et al. im Druck), das für die Gestaltung von Lehr-Lernprozessen in der EB/WB allgemein als zentral betrachtet wird und seit jeher zum Kernbestand der didaktischen Diskussion in der EB/WB gehört (z. B. Arnold et al. 2005; Kraft et al. 2009; Tietgens 1967). Die Bedeutsamkeit dieser Wissensfacette findet Ausdruck in der Häufigkeit, mit der sie in gedruck- 
ten Publikationen (s. Hoffmann 2011) und digitaler Form (z.B. wb-web.de) sowie in einschlägigen Fort- und Weiterbildungen betont wird. Daher bietet sie sich für die Untersuchung der Frage an, ob und inwiefern dieses Wissen mit den verschiedenen genannten ausbildungs- und tätigkeitsbezogenen Merkmalen der Lehrkräfte zusammenhängt.

Diese Frage greift der vorliegende Beitrag auf und bearbeitet damit ein Desiderat in der Erforschung der eingangs vorgestellten Wirkungskette (Terhart 2012); die hier dargestellte Untersuchung stellt zudem einen weiteren Validierungsschritt eines im Projekt ThinK ${ }^{1}$ entwickelten Tests zur Erfassung des Wissens über LehrLernmethoden und -konzepte dar (Abschn. 4.3).

\section{Das Wissen über Lehr-Lernmethoden und -konzepte als Facette des pädagogisch-psychologischen Wissens von Lehrkräften}

Im Rahmen der Forschungen zur professionellen Kompetenz von Lehrkräften in der EB/WB wird das ursprünglich für den Schulkontext entwickelte und dort empirisch bewährte Rahmenmodell der professionellen Kompetenz von Lehrkräften von Baumert und Kunter (2011) vermehrt auch für den Bereich der EB/WB adaptiert (Lencer und Strauch 2016; Marx et al. 2017; Rohs et al. 2017), weil es allgemeine - also fach- und bildungsbereichsübergreifende - und damit auch für die EB/WB relevant erscheinende Bereiche der professionellen Kompetenz thematisiert. Ein weiterer Grund für die Verwendung dieses Modells ist, dass für den Bereich der EB/WB zwar zahlreiche Konzeptualisierungen von Teilbereichen der professionellen Kompetenz von Lehrkräften entwickelt wurden (z. B. Vinepac-Project 2008; Ziep 1990), diesen allerdings kein (gemeinsames) theoretisches Rahmenmodell zugrunde liegt, welches empirisch überprüft wäre (Marx et al. 2014a).

Das Modell der professionellen Kompetenz von Lehrkräften nach Baumert und Kunter (2011) unterscheidet, basierend auf der Kompetenzdefinition von Weinert (2001), in Überzeugungen und Werthaltungen, Selbstregulation und motivationale Orientierungen und integriert die Unterscheidung des professionellen Wissens in Fachwissen, fachdidaktisches Wissen und pädagogisch-psychologisches Wissen (Shulman 1986). Dieses Rahmenmodell liegt auch dem Projekt ThinK zugrunde, in dem das Wissen über Lehr-Lernmethoden und -konzepte als Teil des pädagogischpsychologischen Wissens (PPK) ${ }^{2}$ konzeptualisiert wird und ein Instrument zur fachund bildungsbereichsübergreifenden Erfassung des PPK von Lehrkräften verschiedener Bildungsbereiche entwickelt wird (Marx et al. 2017, 2014b; Voss et al. 2017, 2015). Ziel des Projekts ThinK war es, die Grundlagen zu legen für die Untersuchung

\footnotetext{
1 „Using Digital Media to Assess Generic Aspects of Teachers' Professional Knowledge in Different Educational Contexts" (ThinK) ist ein Projekt-Cluster des WissenschaftsCampus Tübingen, das vom Deutschen Institut für Erwachsenenbildung - Leibniz-Zentrum für Lebenslanges Lernen e. V. in Bonn und dem Hector-Institut für Empirische Bildungsforschung in Tübingen bearbeitet wird.

2 Das PPK, z. B. Lenske et al. (2015) oder Voss et al. (2011), wird häufig auch als pädagogisches Wissen, z. B. Großschedl et al. (2015) oder Voss et al. (2015), und auch als allgemeines pädagogisches Wissen, z. B. König und Blömeke (2012), bezeichnet und ist definiert als Wissen zur Gestaltung und Optimierung von Lehr-Lernsituationen (Voss et al. 2011).
} 
der bislang nicht empirisch geklärten, aber immer wieder aufgegriffenen Frage nach einer „Universaldidaktik“ (s. auch z. B. Aebli 2011; Terhart 2013). ${ }^{3}$ Diese Frage kann aussichtsreich vor allem für das implizit oder explizit als allgemein definierte PPK gestellt werden. Eine Befragung von 44 Wissenschafts- und Praxis-Experten aus und für verschiedene Bildungsbereiche, auch der EB/WB, zeigte hinsichtlich der Einschätzung der PPK-Facette „Wissen über Lehr-Lernmethoden und -konzepte“, dass dieses allseits als bildungsbereichsübergreifend relevant, d.h. als allgemeines Wissen wahrgenommen wird (Marx et al. 2014b); die Annahme erscheint plausibel, dass Schule und EB/WB sich zwar hinsichtlich ihres institutionellen Rahmens unterscheiden und es unterschiedliche Lehrverständnisse geben mag, die personen- oder auch bildungsbereichsspezifisch variieren können. Fokussiert man jedoch das konkrete deklarative und prozedurale Wissen (d.h. nicht die Überzeugungen, Haltungen oder subjektiven Lehrverständnisse) über Lehr-Lernmethoden und -konzepte angesichts konkreter mikrodidaktischer Entscheidungen von Lehrkräften - und so sind die Aufgaben des Tests gestellt -, so ist dieses Wissen generisch konzeptualisierbar und erfassbar (Marx et al. 2017).

\section{Fragestellung und Hypothesen zum Zusammenhang von ausbildungs- und tätigkeitsbezogenen Merkmalen und dem professionellen Wissen von EB/WB-Lehrkräften}

Im Folgenden sollen ausbildungs- und tätigkeitsbezogene Merkmale vorgestellt werden, die daraufhin beleuchtet werden, inwiefern sie im Zusammenhang mit professionellem Wissen, genauer mit dem Wissen über Lehr-Lernmethoden und -konzepte als einer Facette des PPK, stehen. Diese Ausführungen begründen die Hypothesenbildung am Ende jedes Abschnitts und die Fragestellung der Untersuchung: In welchem Zusammenhang stehen die im Folgenden erläuterten ausbildungs- und tätigkeitsbezogenen Merkmale mit dem Wissen über Lehr-Lernmethoden und -konzepte?

\subsection{Merkmal: erziehungs- und bildungswissenschaftliches Studium}

Die berufsbiografisch betrachtet zumeist erste Möglichkeit zur Entwicklung professioneller Lehrkompetenz sind einschlägige erziehungs- und bildungswissenschaftliche Studiengänge, z. B. mit dem Schwerpunkt EB/WB, Wirtschaftspädagogik oder ein Lehramtsstudium mit anschließendem Referendariat. Auf den ersten Blick erscheint es plausibel, dass Absolventinnen und Absolventen solcher Studiengänge, die ca. ein Drittel der Lehrkräfte in der EB/WB ausmachen (Autorengruppe wbpersonalmonitor 2016; Schütz und Nittel 2012), auf eine Lehrtätigkeit durch entsprechende Studieninhalte besser vorbereitet sind als Absolventinnen und Absolventen anderer Studiengänge. Allerdings werden Studierende dieser Studiengänge - abgesehen von den Schullehrkräften - nicht ausschließlich und vornehmlich für

\footnotetext{
${ }^{3}$ Für weitere Lehrkräfte verschiedener Bildungsbereiche betreffende Fragestellungen siehe z. B. Nittel et al. (2011).
} 
eine Lehrtätigkeit ausgebildet, sondern im Falle der EB/WB-Studierenden auch für (die bei hauptberuflich in der EB/WB Beschäftigten zentralen) Tätigkeiten des Weiterbildungsmanagements oder der Programmplanung (Wißhak und Hochholdinger 2016). Dennoch sollte die Auseinandersetzung mit Inhalten der (Allgemeinen) Didaktik, selbst wenn dies nur in grundlegenden Veranstaltungen der Fall ist, aus einer theoretisch-konzeptuellen Perspektive für die Entwicklung des PPK dienlich sein.

Hinweise darauf, dass berufstätige und damit durch erstes und zweites Staatsexamen ausgebildete Schullehrkräfte verglichen mit in der Regel nicht systematisch für eine Lehrtätigkeit ausgebildeten EB/WB-Lehrkräften ein größeres Wissen in Facetten des PPK aufweisen, finden sich bei Marx et al. (2017). Zudem konnte für SchulLehrkräfte gezeigt werden, dass das professionelle Wissen am Ende des Studiums höher war als zu Beginn (Sorge et al. 2017).

Hypothese 1 Vor diesem Hintergrund wird auch für die EB/WB-Lehrkräfte angenommen, dass sich Unterschiede zwischen EB/WB-Lehrkräften mit und ohne erziehungs- und bildungswissenschaftlichem Studium hinsichtlich ihres PPK zeigen, hier operationalisiert anhand des Wissens über Lehr-Lernmethoden und -konzepte: Lehrkräfte mit einem erziehungs- und bildungswissenschaftlichen Studium sollten über mehr Wissen verfügen als Lehrkräfte ohne ein solches Studium.

\subsection{Merkmal: Fort- und Weiterbildungen für Lehrkräfte}

Die Mehrzahl der Lehrkräfte beginnt ohne ein erziehungs- und bildungswissenschaftliches sowie fachdidaktisches Studium ihre Lehrtätigkeit in der EB/WB (Autorengruppe wb-personalmonitor 2016). Für diese Gruppe sind dann - über das Selbststudium und das Beobachtungslernen als Seminarteilnehmende/r hinaus - Fort- und Weiterbildungsmöglichkeiten sowie die konkrete Lehrtätigkeit die ersten Lern- und Kompetenzentwicklungsgelegenheiten hinsichtlich ihrer Lehrtätigkeit.

Fort- und Weiterbildungen für bzw. von Lehrkräfte(n) wird eine grundlegende Bedeutung für die Qualität von Bildungsveranstaltungen in der EB/WB zugeschrieben (von Hippel und Tippelt 2009). Die Vielfalt an Fort- und Weiterbildungen ist groß und reicht vom kollegialen Gespräch bis hin zu zertifizierten Trainerausbildungen (Hohmann 2010). Blickt man auf die Nutzung der non-formalen Angebote für EB/WB-Lehrkräfte, so zeigt sich für das Weiterbildungspersonal, welches im Bereich der Lehre bzw. Training oder Coaching tätig ist, eine Beteiligungsquote an Weiterbildung von 81,8\% (Autorengruppe wb-personalmonitor 2016). Im Durchschnitt nahmen die EB/WB-Lehrkräfte im Zeitraum eines Jahres knapp 55h an Weiterbildungsveranstaltungen teil (Autorengruppe wb-personalmonitor 2016), wobei allerdings unklar ist, welche Inhalte in diesen thematisiert wurden und ob diese überhaupt auf die Lehrtätigkeit bezogen sind. Legt man jedenfalls Befragungsergebnisse zum Fortbildungsbedarf von Lehrkräften zugrunde, wie sie z. B. im Projekt KomWeit durchgeführt wurden, dann zeigt sich auch hier, dass „Didaktik/Methodik“ sowohl vom planend-disponierenden Weiterbildungspersonal als auch von Lehrkräften für besonders zentral erachtet werden (Fuchs et al. 2009). 
Fort- und Weiterbildungsangebote für EB/WB-Lehrkräfte zum Thema Lehren und Lernen/Methodik/Didaktik sind in der Regel spezifischer auf die Lehrtätigkeit und die Bedürfnisse der Lehrkräfte in der EB/WB ausgerichtet, als dies die Mehrzahl der erziehungs- und bildungswissenschaftlichen Studiengänge sein können (Wißhak 2017). Zudem liegen empirische Befunde vor, die zeigen, dass einschlägige Fortbildungen das Wissen und die Kognitionen von Lehrkräften positiv verändern können (Digel 2013; Goeze 2016).

Hypothese 2 Für das Wissen über Lehr-Lernmethoden und -konzepte wird ein positiver Zusammenhang mit Fort- und Weiterbildungen angenommen: Je mehr Stunden an spezifischer Fort- und Weiterbildung zum Thema Lehren und Lernen/ Methodik/Didaktik absolviert wurden, über desto mehr Wissen zu Lehr-Lernmethoden und -konzepten verfügen EB/WB-Lehrkräfte.

\subsection{Merkmale: Lehrerfahrung und Lehrumfang}

Von praktischen Lehrerfahrungen wird angenommen, dass sie beim Erwerb professioneller Kompetenz, die sich auch aus Erfahrungswissen speist, eine wichtige Rolle spielen (Voss et al. 2011). Eigene Lehrerfahrungen sind die Voraussetzung dafür, dass das z. B. in Fort- und Weiterbildungen oder im Selbststudium erworbene theoretisch-konzeptuelle Wissen angewendet werden kann und nicht träge bleibt (grundlegend z. B. Gruber et al. 2000). Insgesamt liegen bislang nur wenige Studien vor, die den Einfluss der Lehrerfahrung auf die professionelle Kompetenz untersucht haben (Voss et al. 2015) oder gar der Bedeutung der Lehrerfahrung für den Lernfortschritt der Lernenden (Rivkin et al. 2005). Dieses Erfahrungswissen dürfte bei längerer Lehrerfahrung bzw. bei höherem aktuellen Lehrumfang zu mehr Auseinandersetzungen mit Lehr-Lernmethoden und -konzepten und damit zu mehr informell erworbenem Wissen in diesem Bereich geführt haben.

Hypothesen 3a und 3b Für den aktuellen Lehrumfang in Wochenstunden (H3a) sowie die Lehrerfahrung in Jahren (H3b) wird daher ein positiver Zusammenhang zum Wissen über Lehr-Lernmethoden und -konzepte angenommen: Je höher der aktuelle Lehrumfang in Wochenstunden bzw. je mehr Lehrerfahrung in Jahren, desto mehr Wissen über Lehr-Lernmethoden und -konzepte zeigt sich bei den EB/WBLehrkräften.

Während die Ausbildung von Schullehrkräften mittlerweile zunehmend mehr Praxisphasen vorsieht, deren Verzahnung mit „Theoriephasen“ als wünschenswert, weil vielversprechend, gilt (so z. B. European Commission 2015), wird die Verzahnung von Theorie und Praxis bei Lehrkräften in der EB/WB häufig berufsbegleitend realisiert: einerseits durch die praktische Lehrtätigkeit und andererseits durch den Besuch einschlägiger Fort- und Weiterbildungen. Im Vergleich zur Ausbildung von Schullehrkräften fehlt Lehrkräften in der EB/WB häufig weniger die praktische Lehrerfahrung, sondern eher die theoretisch-konzeptuelle Wissensgrundlage zu didaktischen Konzepten und Prinzipien des Lehrens und Lernens. 
Hypothese 4 Vor diesem Hintergrund wird ein Interaktionseffekt zwischen Lehrerfahrung und dem Besuch einschlägiger Fort- und Weiterbildungen erwartet: Lehrkräfte mit wenig Lehrerfahrung dürften stärker von zusätzlichen Stunden absolvierter Fort- und Weiterbildung hinsichtlich des Wissens profitieren als Lehrkräfte mit viel Lehrerfahrung. Für Lehrkräfte mit weniger Lehrerfahrung in Jahren wird also ein stärkerer Zusammenhang zwischen den absolvierten Stunden an Fort- und Weiterbildung und dem Wissen über Lehr-Lernmethoden und -konzepten erwartet als für Lehrkräfte mit mehr Lehrerfahrung.

Der angenommene Interaktionseffekt impliziert auch, dass Lehrkräfte, die weniger Stunden an Fort- und Weiterbildung absolviert haben, hinsichtlich ihres Wissens stärker von zusätzlichen Jahren an Lehrerfahrung profitieren als Lehrkräfte, die viele Stunden an Fort- und Weiterbildung absolviert haben: Für Lehrkräfte mit weniger absolvierten Fort- und Weiterbildungsstunden wird ein stärkerer Zusammenhang zwischen den Jahren an Lehrerfahrung und dem Wissen über Lehr-Lernmethoden und -konzepten erwartet als für Lehrkräfte mit mehr absolvierten Stunden an Fortund Weiterbildung.

\subsection{Merkmal: Schwerpunkt der Lehrtätigkeit in der allgemeinen Erwachsenenbildung oder in der beruflich-betrieblichen Weiterbildung}

Ein weiterer Einfluss auf die Entwicklung der professionellen Kompetenz der Lehrkräfte kann deren Tätigkeitsschwerpunkt sein. Eine gängige Typologie zur Unterscheidung von Bildungsangeboten differenziert nach der inhaltlichen Ausrichtung der Bildungsangebote in allgemeine Erwachsenenbildung und beruflich-betriebliche Weiterbildung, wie sie z.B. im wb-personalmonitor vorgenommen wurde (Autorengruppe wb-personalmonitor 2016).

Die Erwartungshaltung der Teilnehmenden in der beruflich-betrieblichen WB an das lehr-lernmethodische Wissen der Lehrkräfte angesichts des eigenen potenziellen „Konsequenzenreichtums“ bei möglichem ausbleibenden Lernerfolg im beruflichen Kontext sowie die höhere finanzielle Vergütung der Trainerinnen und Trainer in der beruflich-betrieblichen WB (im Vergleich zur allgemeinen EB) könnten eine höhere „Anspruchshaltung“ der Teilnehmenden in diesem Weiterbildungsbereich begründen. Die Folge könnte sein, dass die Trainerinnen und Trainer sich stärker selbstständig mit methodischem und didaktischem Wissen auseinandersetzen, um die LehrLernveranstaltungen zur Zufriedenheit der Teilnehmenden gestalten zu können. Dies scheint insbesondere dann plausibel, wenn die Teilnehmerzufriedenheit Geschäftsund Existenzgrundlage ist für die Trainerinnen und Trainer, wie es in der beruflichbetrieblichen WB häufig der Fall ist im Vergleich zur den meist nebenberuflich ausgeübten Lehrtätigkeiten in der allgemeinen EB. Insofern könnten die allgemeine EB und die beruflich-betriebliche WB „differenzielle Entwicklungsmilieus“ (Neumann et al. 2007) für das Lehrkräfte-Wissen darstellen.

Hypothese 5 Vor diesem Hintergrund wird folgender Unterschied angenommen: Im Schwerpunkt in der beruflich-betrieblichen WB tätige Lehrkräfte verfügen über 
mehr Wissen über Lehr-Lernmethoden und -konzepte als schwerpunktmäßig in der allgemeinen EB tätige Lehrkräfte.

\section{Methodisches Vorgehen}

\subsection{Stichprobe}

An der Hauptstudie des Projekts ThinK nahmen insgesamt $N=212$ EB/WB-Lehrkräfte teil, dabei handelte es sich um eine ad-hoc-Stichprobe. Für den vorliegenden Beitrag wurden drei Fälle aufgrund von extremen Ausreißern in den Prädiktorvariablen ausgeschlossen, so dass für die nachfolgenden Analysen $N=209$ EB/WBLehrkräfte zur Verfügung standen. Um Personen mit fehlenden Werten auf einzelnen Variablen nicht aus den Analysen ausschließen zu müssen, wurde in Mplus (Muthén und Muthén 2015) die Funktion full information maximum likelihood (FIML) verwendet, da ein listenweiser Fallausschluss neben einem Verlust an Effizienz bei der Parameterschätzung auch zu fehlerhaften Befunden führen kann (Lüdtke et al. 2007). Die Lehrkräfte im Alter von 24-77 Jahren $(M=48,39 ; S D=12,19)$ verfügten zum Erhebungszeitpunkt über eine Lehrerfahrung zwischen 1 und 50 Jahren $(M=12,70$; $S D=10,44)$ und lehrten pro Woche zwischen 1 und $50 \mathrm{~h}(M=11,97 ; S D=11,06)$. Der Anteil an Akademikern (73,54\%), die mehrheitlich nebenberufliche Beschäftigung $(63,02 \%)$ bei mehreren Bildungseinrichtungen, der Anteil an weiblichen Lehrkräften $(48,44 \%)$ sowie der Anteil an Personen mit einem erziehungs- und bildungswissenschaftlichen Studium $(25,84 \%)$ weisen die vorliegende Stichprobe als typisch für die EB/WB aus (Autorengruppe wb-personalmonitor 2016). Die Lehrkräfte nahmen insgesamt zwischen 0 und $520 \mathrm{~h}$ an Weiterbildungen zum Thema Lehren und Lernen/Methodik/Didaktik teil $(M=95,85 ; S D=134,18)$. Der Anteil der Lehrkräfte in der allgemeinen EB und der beruflich-betrieblichen WB war nahezu ausgewogen (EB: 46,32\%). Nach den ausdifferenziert abgefragten Themenschwerpunkten (z.B. IT mit berufs- oder nicht berufsbezogenem Schwerpunkt) konnten die Lehrkräfte den beiden Bereichen zugeordnet werden.

\subsection{Erfassung des Wissens über Lehr-Lernmethoden und -konzepte: Aufgaben}

Das Wissen über Lehr-Lernmethoden und -konzepte wurde im Projekt ThinK durch (vorläufig) fünf Aufgaben erfasst (Marx et al. o.J.). Diese Aufgaben lagen im gleichen Aufgabenformat vor wie die Beispielaufgabe in Tab. 1 und umfassen bis zu fünf Antwortoptionen im Richtig-falsch-Antwortformat. Die Richtig-falsch-Antworten hatten einen gemeinsamen Aufgabenstamm und ähnelten damit Testlets (Wainer und Kiely 1987).

Die fünf Aufgaben hatten ihren inhaltlichen Schwerpunkt auf fünf verschiedenen lehr-lernmethodischen Vorgehensweisen. Eine Aufgabe (A) thematisierte die Anstrengungsbereitschaft bei Gruppenarbeiten, (B) die Vortragsmethode (Tab. 1), (C) die Vermeidung aufgabenbedingter Trittbrettfahrereffekte bei Gruppenarbeiten, (D) die Feedback-Methode und (E) die Aktivierung von Vorwissen in Lehr-Lernsituationen. 
Tab. 1 Beispiel-Aufgabe zur Erfassung des Wissens über Lehr-Lernmethoden und -konzepte

\begin{tabular}{|c|c|c|}
\hline \multicolumn{3}{|c|}{$\begin{array}{l}\text { Bitte beurteilen Sie, welche der folgenden Aussagen stimmen oder nicht stimmen } \\
\text { Kreuzen Sie bitte in jeder Zeile ein Kästchen an }\end{array}$} \\
\hline $\begin{array}{l}\text { Der Lehrervortrag (auch als Vortragsmethode bezeichnet) ist dann } \\
\text { geeignet, wenn ... }\end{array}$ & $\begin{array}{l}\text { Stimmt } \\
\text { nicht }\end{array}$ & Stimmt \\
\hline ... das Hauptziel in der Vermittlung von Informationen besteht & $\square$ & $\square$ \\
\hline ... eine Einführung in einen bestimmten Bereich gegeben werden soll & $\square$ & $\square$ \\
\hline ... das Behalten des Stoffs über einen längeren Zeitraum erwünscht ist & $\square$ & $\square$ \\
\hline $\begin{array}{l}\text {... die Beteiligung der Lernenden eine wesentliche Voraussetzung ist, um } \\
\text { ein Lernziel zu erreichen }\end{array}$ & $\square$ & $\square$ \\
\hline $\begin{array}{l}\text {... der Stoff für die Lernenden komplex ist oder eine Menge Details ent- } \\
\text { hält }\end{array}$ & $\square$ & $\square$ \\
\hline
\end{tabular}

\subsection{Erfassung des Wissens über Lehr-Lernmethoden und -konzepte: Vorbereitende Validierungsschritte}

Da die fünf Aufgaben aus theoretisch-konzeptueller Perspektive zwar einerseits alle der Wissensfacette „Wissen über Lehr-Lernmethoden und -konzepte“ zuzuordnen sind und damit allen Aufgabe gemeinsames Wissen zu ihrer Beantwortung erfordern, andererseits aber jeweils einen spezifischen Themenschwerpunkt setzen, stellt sich die Frage nach der Anzahl der Wissensdimensionen, die für die Beantwortung der Aufgaben erforderlich sind. Diese Frage wurde an anderer Stelle gesondert untersucht (Marx et al. o.J.), so dass hier von einer erprobten Struktur ausgegangen werden kann. Ein Rasch-Testlet-Modell (Wang und Wilson 2005) erklärte das Antwortverhalten der EB/WB-Lehrkräfte am besten, das die beschriebene Dimensionalitätssstruktur mit gemeinsamem Wissen über verschiedene Aufgaben hinweg sowie spezifischem Wissen für die jeweilig einzelne Aufgabe berücksichtigt (Abb. 1a). Allerdings stellt sich die Frage, ob v.a. die modellierten aufgabenspezifischen Wissensdimensionen nicht letztlich konstruktirrelevante Varianz abbilden, also beispielsweise innerhalb von Personen über die Zeit hinweg inkonsistente Merkmale, die z.B. auf die konkrete Befragungssituation zurückzuführen sind oder auf den Test als solchen und damit letztlich Effekte der Methode der Erfassung darstellen (z.B. Kelava und Schermelleh-Engel 2012). Daher wurde für den vorliegenden Beitrag die in Marx et al. (o.J.) vorgenommene Modellierung einem weiteren Validierungsschritt unterzogen, der hier nur zusammenfassend skizziert werden kann. Für diesen Validierungsschritt stellt sich die Frage, ob sich für die insgesamt sechs (eine gemeinsame $(\mathrm{g})$ und fünf spezifische $(\mathrm{A}-\mathrm{E})$ ) Wissensdimensionen jeweils ein aus theoretisch-konzeptueller Sicht plausibles Zusammenhangsmuster mit den oben genannten ausbildungs- und tätigkeitsbezogenen Merkmalen zeigt.

Das in Marx et al. (o.J.) vorgestellte Modell wurde in einem ersten Schritt in Mplus Version 7.4 spezifiziert. ${ }^{4}$ In einem zweiten Schritt wurde pro Wissensdimension durch eine latente multiple Regressionsanalyse auf die ausbildungs- und tä-

\footnotetext{
4 Gleiche Faktorladungen für alle Indikatoren auf die allen Aufgaben gemeinsame Wissensdimension und gleiche Faktorladungen der jeweils spezifischen Indikatoren auf die jeweils spezifischen Wissensdimensionen; die Varianz aller Wissensdimensionen wurde auf eins fixiert, s. zur Spezifikation von Rasch-Modellen in Mplus Muthén und Muthén (1998-2015).
} 
Modell (a)

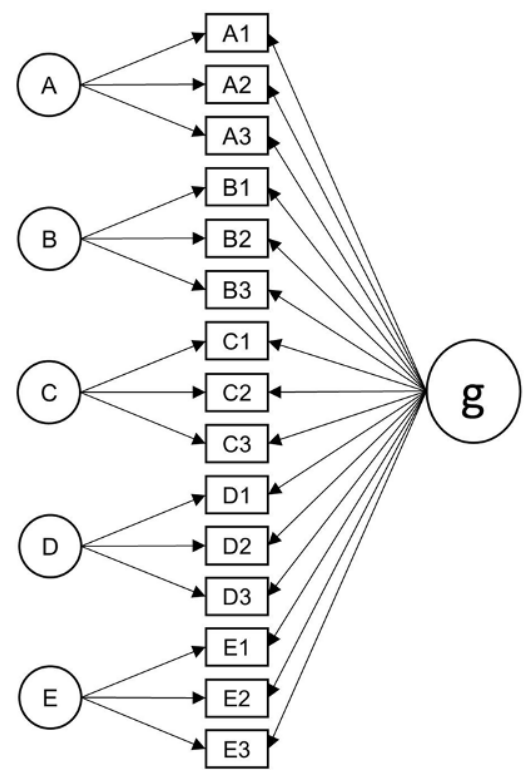

Modell (b)

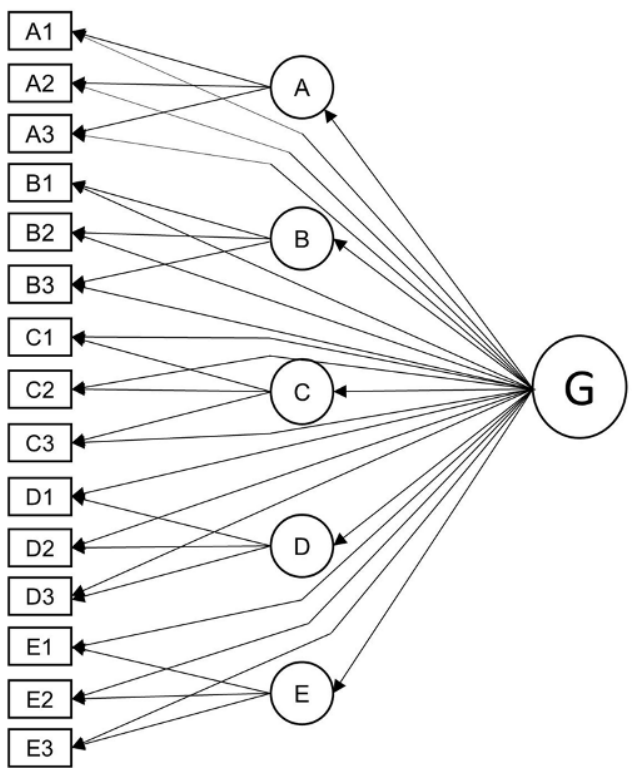

Abb. 1 a Rasch-Testlet-Modell (nach Wang und Wilson 2005). b Second-order-Modell (nach Chen et al. 2006)

tigkeitsbezogenen Merkmale regressiert (die vorher auf Konfundierungen überprüft wurden, s. Abschn. 5). Es zeigte sich cum grano salis über alle sechs Wissensdimensionen hinweg ein weitgehend plausibles Zusammenhangsmuster (Tab. 4 im Anhang). Hierdurch eröffnen sich zwei Möglichkeiten - vorläufig bzw. solange keine weiteren Validierungsstudien dagegen sprechen: Erstens können die Zusammenhänge zwischen ausbildungs- und tätigkeitsbezogenen Merkmalen einerseits und dem Wissen über Lehr-Lernmethoden und -konzepte andererseits als ebensolche Zusammenhänge aus einer theoretisch-konzeptuellen Perspektive interpretiert werden; zweitens können die insgesamt sechs Wissensdimensionen des spezifizierten Rasch-Testlet-Modells in einem gemeinsamen Faktor (G) in einem full secondorder-Modell zusammengefasst werden, der zwar hinsichtlich (etwaiger) verschiedener Wissensbereiche ein weniger differenzierbares, aber überschaubareres Bild der Zusammenhänge anbieten kann (Abb. 1b). Dieser gemeinsame Faktor G bildet im Ergebnisbericht das Wissen über Lehr-Lernmethoden und -konzepte ab. Das full second-order-Modell wurde spezifiziert wie von Chen et al. (2006) vorgeschlagen. Bei diesem Vorgehen zeigte sich für die Erfassung des Wissens über LehrLernmethoden und -konzepte eine Reliabilität von $\omega t=0,76$ (Marx et al. o.J.).

Die Erfassung der ausbildungs- und tätigkeitsbezogenen Merkmale wurde mit Instrumenten aus den Erhebungen des wb-personalmonitors durchgeführt (Autorengruppe wb-personalmonitor 2016). Die formulierten Hypothesen werden unter Berücksichtigung der Kontrollvariablen Alter und Geschlecht überprüft. Der Diskussion des Beitrags vorgreifend soll an dieser Stelle bereits betont werden, dass aufgrund der für die zugrundeliegenden Analyseverfahren kleinen und ausgewähl- 
Tab. 2 Korrelationsmatrix der Variablen Alter, Lehrerfahrung in Jahren, Stunden Fort- und Weiterbildung in Lehren und Lernen/Methodik/Didaktik und aktueller Lehrumfang in Wochenstunden

\begin{tabular}{llll}
\hline & Alter & $\begin{array}{l}\text { Lehrerfahrung in } \\
\text { Jahren }\end{array}$ & $\begin{array}{l}\text { Stunden Fort- und } \\
\text { Weiterbildung }\end{array}$ \\
\hline Lehrerfahrung in Jahren & $\begin{array}{l}r=0,560^{*} \\
(0,119)\end{array}$ & - & - \\
Stunden Fort- und Wei- & $r=0,170 \mathrm{n} . \mathrm{s}$. & $\begin{array}{l}r=0,365 \mathrm{n} . \mathrm{s} . \\
(0,353)\end{array}$ & - \\
terbildung & $(0,270)$ & $r=0,197 \mathrm{n} . \mathrm{s}$. & $r=0,225 \mathrm{n} . \mathrm{s}$. \\
Aktueller Lehrumfang in & $r=0,001 \mathrm{n} . \mathrm{s}$. & $(0,114)$ & $(0,117)$ \\
Wochenstunden & $(0,132)$ &
\end{tabular}

Werte in Klammern: Standardfehler

$r$ Pearson Korrelation

n. s. $p>0,05$

$* \mathrm{p} \leq 0,05$

ten Stichprobengröße der vorliegende Beitrag trotz der Überprüfung von Hypothesen einen inhaltlich explorierenden Ansatz verfolgt und nicht den Anspruch erhebt, repräsentativ-generalisierbare Aussagen zu treffen.

\section{Ergebnisse}

In einem ersten Schritt, und damit dem oben berichteten Validierungsschritt vorangehend, wurden die Prädiktorvariablen auf Konfundierungen überprüft. Hierbei zeigte sich ein erwartbarer signifikanter Zusammenhang zwischen den Variablen Alter und Lehrerfahrung in Jahren (Tab. 2).

Auch zeigte sich ein signifikanter Zusammenhang zwischen den Variablen erziehungs- und bildungswissenschaftliches Studium und Geschlecht, wobei mehr Frauen $(n=35)$ als Männer $(n=19)$ ein erziehungs- und bildungswissenschaftliches Studium absolviert haben bzw. weniger Frauen $(n=64)$ als Männer $(n=74)$ kein erziehungs- und bildungswissenschaftliches Studium absolviert haben; $x^{2}(1)=4,571$; $p=0,033$. Zudem zeigte sich ein signifikanter Zusammenhang zwischen den Variablen Geschlecht und Tätigkeitsschwerpunkt in der EB/WB, wobei mehr Frauen $(n=65)$ als Männer $(n=37)$ in der EB und mehr Männer $(n=56)$ als Frauen $(n=29)$ in der WB arbeiteten; $x^{2}(1)=15,095 ; p=0,000$. Für Lehrkräfte in der allgemeinen EB $(M=51,56 ; S D=11,46)$ zeigte sich ein signifikant höheres Durchschnittsalter als für Lehrkräfte in der beruflich-betrieblichen WB $(M=44,42$; $S D=11,84) ;\left(t(188)=4,216 ; p=0,000 ; \mathrm{g}_{\text {Hedges }}=-0,614\right)$. Lehrkräfte ohne erziehungsund bildungswissenschaftliches Studium $(M=11,67, S D=9,75)$ verfügten über eine signifikant geringere Lehrerfahrung als Lehrkräfte mit erziehungs- und bildungswissenschaftlichem Studium $(M=15,38 ; S D=11,74) ;(t(190)=-2,219 ; p=0,028$; $\left.\mathrm{g}_{\text {Hedges }}=-0,360\right)$. Für die übrigen Prädiktorvariablen zeigten sich keine bedeutsamen Zusammenhänge.

Um die Frage zu beantworten, in welchem Zusammenhang die ausbildungs- und tätigkeitsbezogenen Merkmale von Lehrkräften aus der EB/WB mit ihrem Wissen über Lehr-Lernmethoden und -konzepte stehen, wurde zur Modellierung dieses Wissens ein full second-order-Modell spezifiziert (Chen et al. 2006 und Abb. 1b). 
Tab. 3 Ergebnisse der multiplen linearen Regressionsanalyse zur Vorhersage des „Wissens über LehrLernmethoden und -konzepte" von Lehrkräften aus der Erwachsenen- und Weiterbildung aus ausbildungsund tätigkeitsbezogenen Merkmalen $(N=209)$

\begin{tabular}{|c|c|c|c|c|c|c|}
\hline & $B$ & $S E$ & $\beta$ & $S E$ & $t$ & $p$ \\
\hline Alter & $-0,017$ & 0,011 & $-0,413$ & 0,161 & $-2,568$ & 0,010 \\
\hline Geschlecht & $-0,130$ & 0,170 & $-0,132$ & 0,154 & $-0,859$ & 0,391 \\
\hline $\begin{array}{l}\text { Tätigkeitsschwerpunkt EB oder } \\
\text { WB }\end{array}$ & 0,414 & 0,227 & 0,420 & 0,147 & 2,851 & 0,004 \\
\hline $\begin{array}{l}\text { Aktueller Lehrumfang in Wochen- } \\
\text { stunden }\end{array}$ & $-0,004$ & 0,007 & $-0,087$ & 0,153 & $-0,567$ & 0,571 \\
\hline $\begin{array}{l}\text { Erziehungs- und bildungswissen- } \\
\text { schaftliches Studium }\end{array}$ & $-0,051$ & 0,157 & $-0,045$ & 0,138 & $-0,328$ & 0,743 \\
\hline Lehrerfahrung in Jahren & $-0,027$ & 0,082 & $-0,055$ & 0,170 & $-0,321$ & 0,748 \\
\hline Stunden Fort- und Weiterbildung & 0,214 & 0,144 & 0,438 & 0,208 & 2,110 & 0,035 \\
\hline $\begin{array}{l}\text { Interaktion Lehrerfahrung und } \\
\text { Fort- und Weiterbildung }\end{array}$ & $-0,188$ & 0,123 & $-0,452$ & 0,221 & $-2,040$ & 0,041 \\
\hline Gesamtmodell: $R^{2}=62,6 \%$ & & & & & & \\
\hline
\end{tabular}

Geschlecht: Frauen $=0$, Männer $=1$

EB/WB: Tätigkeitsschwerpunkt in der allgemeinen Erwachsenenbildung $=0$, Tätigkeitsschwerpunkt in der beruflich-betrieblichen Weiterbildung $=1$;

Erziehungs- und bildungswissenschaftliches Studium: $\operatorname{nein}=0, \mathrm{ja}=1$

Insgesamt wurden 90 Modellparameter frei geschätzt (ESTIMATOR = MLF, Muthén und Muthén 2015). Alle dichotomen Variablen (Geschlecht, Schwerpunkt EB/WB, erziehungs- und bildungswissenschaftliches Studium) wurden als Dummy-Variablen in die Analysen aufgenommen. In Tab. 3 werden die unstandardisierten ${ }^{5}$ und die standardisierten Regressionsgewichte berichtet, Standardfehler, $t$-Wert und $p$-Wert beziehen sich auf die standardisierten Regressionsgewichte (STANDARDIZED (STDY), Muthén und Muthén 2015).

Alter stellte eine Kontrollvariable dar, für die sich ein signifikanter negativer Zusammenhang mit dem Wissen über Lehr-Lernmethoden und -konzepte zeigte. Es zeigten sich - entgegen der formulierten Hypothese 3a - keine signifikanten Zusammenhänge zwischen dem Wissen über Lehr-Lernmethoden und -konzepte und dem aktuellen Lehrumfang in Wochenstunden.

Das Ausmaß an diesem Wissen scheint zudem wider Erwarten in keinem statistisch bedeutsamen Zusammenhang damit zu stehen, ob die bisherige Lehrerfahrung kurz oder lang ist (H3b) und ob ein erziehungs- und bildungswissenschaftliches Studium absolviert wurde oder nicht (H1). Konform zu den Hypothesen 2 und 4 zeigten sich zum Wissen über Lehr-Lernmethoden und -konzepte ein signifikanter positiver Zusammenhang zwischen der Anzahl der Stunden in Fort- und Weiterbildung (H2) sowie ein signifikanter negativer Interaktionseffekt zwischen Lehrerfahrung in Jahren und den absolvierten Stunden an Fort- und Weiterbildung (H4). Um den Interaktionseffekt besser interpretieren zu können, sind in Abb. 2 für die Gruppe der Lehrkräfte mit einer Standardabweichung unter dem Durchschnitt $(B=0,402)$,

\footnotetext{
${ }_{5}$ Die Variablen absolvierte Stunden an Fort- und Weiterbildung und Lehrerfahrung in Jahren wurden aufgrund der Schiefe der Verteilungen z-standardisiert.
} 


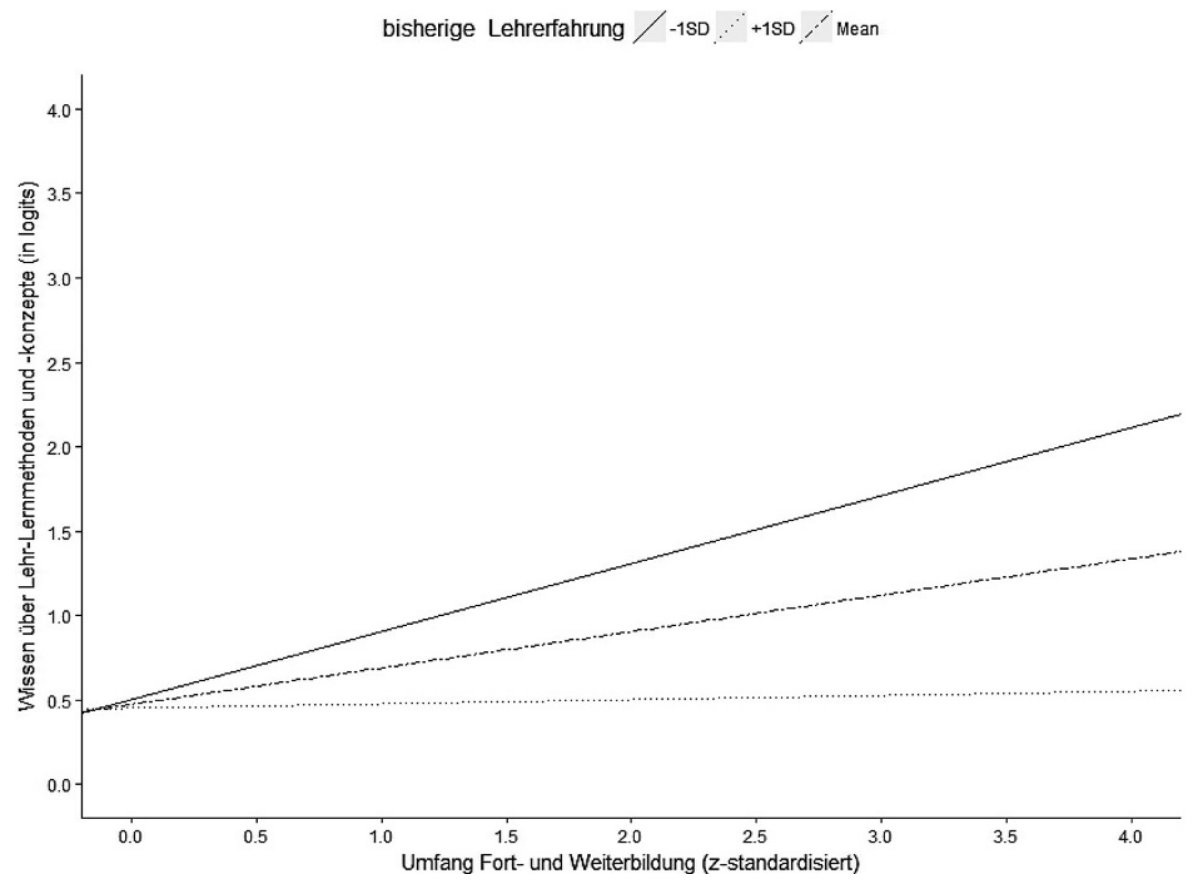

Abb. 2 Interaktionseffekt zwischen Lehrerfahrung in Jahren und den absolvierten Stunden in Fort- und Weiterbildung

Durchschnitt $(B=0,214)$ und einer Standardabweichung über dem Durchschnitt $(B=0,026)$ an Lehrerfahrung in Jahren die Regressionsgeraden des Wissens über Lehr-Lernmethoden und -konzepte für den absolvierten Umfang an Fort- und Weiterbildung abgebildet. Im vorliegenden Beitrag wurde der Interaktionseffekt mit der Variablen Lehrerfahrung in Jahren als Moderatorvariable abgebildet. Für Lehrkräfte mit einer Standardabweichung über dem Durchschnitt an Lehrerfahrung zeigte sich ein weniger enger Zusammenhang zwischen dem absolvierten Umfang an Fortund Weiterbildung zum Wissen über Lehr-Lernmethoden und -konzepte als für die Gruppe, die durchschnittlich bzw. wenig Lehrerfahrung hat. Dies bedeutet, dass für Lehrkräfte mit wenig Lehrerfahrung der Umfang an absolvierter Fort- und Weiterbildung einen größeren Effekt zu haben scheint als für Lehrkräfte mit durchschnittlicher Lehrerfahrung oder einer Standardabweichung unter dem Durchschnitt - Lehrkräfte mit wenig Lehrerfahrung scheinen also stärker von Fort- und Weiterbildung zu profitieren als Lehrkräfte mit durchschnittlicher oder viel Lehrerfahrung. Bei Abbildung der Variablen Umfang an Fort- und Weiterbildung als Moderatorvariable zeigte sich u. a. für die Lehrkräfte mit weniger Fort- und Weiterbildung ein stärkerer positiver Zusammenhang zwischen Lehrerfahrung und dem Wissen über Lehr-Lernmethoden und -konzepte als für Lehrkräfte mit durchschnittlichem Umfang an Fort- und Weiterbildung (Abb. 3 im Anhang). 
Konform zur Hypothese 5 zeigt sich ein signifikanter Unterschied im Wissen über Lehr-Lernmethoden und -konzepte zugunsten der Lehrkräfte mit Schwerpunkt in der beruflich-betrieblichen WB gegenüber Lehrkräften in der allgemeinen EB. Für das gesamte Modell zeigte sich eine Varianzaufklärung im Wissen über LehrLernmethoden und -konzepte von $62,6 \%$.

\section{Zusammenfassung und Diskussion der Ergebnisse}

Dieser Beitrag widmete sich der für den Bereich der EB/WB bislang unbeantworteten Frage, welche Zusammenhänge sich zwischen ausbildungs- und tätigkeitsbezogenen Merkmalen und dem Wissen über Lehr-Lernmethoden und -konzepte von EB/WB-Lehrkräften zeigen. Die beobachteten Zusammenhänge werden nachfolgend vor dem Hintergrund der für diesen Beitrag zur Verfügung stehenden Stichprobe kritisch reflektiert und in ihrer Bedeutsamkeit eingeordnet.

Es zeigten sich keine Unterschiede zwischen EB/WB-Lehrkräften mit und ohne erziehungs- und bildungswissenschaftlichem Studium im Wissen über Lehr-Lernmethoden und -konzepte, aber Unterschiede in Abhängigkeit vom Umfang an absolvierten Stunden an Fort- und Weiterbildungen zum Thema Lehren und Lernen/ Methodik/Didaktik. Diese Resultate können als Konsequenz der empirischen Befundlage gedeutet werden, ,dass Trainerausbildungen und erziehungswissenschaftliche Studiengänge recht unterschiedliche Inhalte vorsehen“ (Wißhak 2017): Während einschlägige Fortbildungen einen direkten Mehrwert für das Wissen über LehrLernmethoden und -konzepte intendieren und auch zu haben scheinen (wenn man diese kausale Wirkrichtung unterstellen mag), erbringen erziehungs- und bildungswissenschaftliche Studiengänge keinen hier belegbaren Mehrwert für dieses Wissen. Letzteres Ergebnis sollte allerdings vorsichtig interpretiert werden. Denn die vergleichsweise undifferenzierte Abbildung der Lehrkräfte mit unterschiedlichen erziehungs- und bildungswissenschaftlichen Studienabschlüssen in einer gemeinsamen Gruppe kann dazu führen, dass sich etwaige doch vorhandene Effekte ggf. verlieren. Die aus theoretisch-konzeptueller Perspektive plausibel erscheinende Annahme, dass sich (wenigstens bestimmte) erziehungs- und bildungswissenschaftliche Studiengänge positiv - ggf. aber weniger stark als einschlägige Fort- und Weiterbildung - auf das Wissen über Lehr-Lernmethoden und -konzepte auswirken könnten, wird daher trotz der Hinweise, die der vorliegende Beitrag gibt, nicht vollständig aufgegeben, sondern sie bedarf weiterer empirischer Klärung.

Dass mehr Stunden an spezifischer Fort- und Weiterbildung zum Thema Lehren und Lernen/Methodik/Didaktik mit mehr Wissen zu Lehr-Lernmethoden und -konzepten einhergehen und dass insbesondere weniger Lehrerfahrene, wenn sie umfangreichere Fort- und Weiterbildungen besucht haben, über mehr methodisches Wissen verfügen, darf als ermutigender Befund gedeutet werden: Die eingangs angesprochenen vielfältigen Initiativen zur Fort- und Weiterbildung von (angehenden) Lehrkräften setzen an der richtigen Stelle an, und für den Test darf das Ergebnis - angesichts einer positiven Korrelation mit diesem sehr einschlägigen Außenkriterium - als weiterer Hinweis auf eine valide Erfassung gewertet werden. 
Für die Lehrkräfte mit vergleichsweise wenig Fort- und Weiterbildung zeigte sich ein stärkerer positiver Zusammenhang zwischen Lehrerfahrung und dem Wissen über Lehr-Lernmethoden und -konzepte als für Lehrkräfte mit durchschnittlicher Fort- und Weiterbildung (Abb. 3 im Anhang). Vor dem Hintergrund der Annahme, dass mit einem höheren Wissen über Lehr-Lernmethoden und -konzepte eine gröBere Chance auf eine höhere Unterrichtsqualität einhergeht, kann eine praktische Implikation dieses Befunds sein, dass es sich trotz der „kompensierenden“ Wirkung von Lehrerfahrung lohnt, zu einem frühen Zeitpunkt, ggf. noch vor Beginn der Lehrtätigkeit, in die Ausbildung der EB/WB-Lehrkräfte zu investieren. Denn interessant ist, dass sich kein direkter Zusammenhang zwischen der Lehrerfahrung, erfasst durch den aktuellen Lehrumfang pro Woche in den vergangenen zwölf Monaten, bzw. der bisherigen Lehrerfahrung insgesamt, erfasst in Jahren, mit dem Wissen über Lehr-Lernmethoden und -konzepte zeigt. Lehrkräfte werden also weder durch das kurz- noch durch das längerfristige Anhäufen von Lehrerfahrung (wobei offenbleiben muss, in welchem Gesamtvolumen) kenntnisreicher bezogen auf LehrLernmethoden und -konzepte (ähnlich Rivkin et al. 2005). Dieser Befund relativiert die praktische Bedeutung von ,,viel Erfahrung“ einer Lehrkraft z. B. für die Trainerauswahl - immer vorausgesetzt, dass methodische Kenntnis und praktisches Lehrhandeln nicht völlig unabhängig voneinander sind.

Ein erwartungskonform signifikanter Unterschied im Wissen über Lehr-Lernmethoden und -konzepte zeigte sich zugunsten der Lehrkräfte mit Arbeitsschwerpunkt in der beruflich-betrieblichen Weiterbildung gegenüber Lehrkräften in der allgemeinen Erwachsenenbildung. Ob die hypothesenleitende Annahme zutrifft, dass eine ausgeprägtere ,Anspruchshaltung“ der Teilnehmenden an Methodenvielfalt und didaktische Professionalität in ersterem Weiterbildungsbereich größer ist (sowie üblicherweise eine höhere existenzielle Abhängigkeit der Trainer von der Zufriedenheit der „Kunden“, weil in der betrieblichen Weiterbildung mehr Hauptberuflichkeit der Trainer vorherrscht) als im Letzteren und dies „differenzielle Entwicklungsmilieus“ für das Lehrkräfte-Wissen begründet, kann hier in einer korrelativen Studie nicht als belegt gewertet werden, es liegt jedoch nahe. Der berichtete Befund kann als Anregung dazu dienen, im Rahmen zukünftiger Untersuchungen das Merkmal des Tätigkeitsschwerpunkts - und wenn möglich auch des Themenschwerpunkts - differenzierter zu betrachten.

Überraschend zeigte sich für die Kontrollvariable Alter ein negativer Zusammenhang zum Wissen über Lehr-Lernmethoden und -konzepte. Hier könnte es jedoch aufgrund der Stichprobengröße zu einer Konfundierung von Alter mit Lehrerfahrung gekommen sein (Tab. 2).

Die ausbildungs- und tätigkeitsbezogenen Merkmale im aufgestellten Regressionsmodell erklären insgesamt ungefähr zwei Drittel der Unterschiede im Wissen über Lehr-Lernmethoden und -konzepte der EB/WB-Lehrkräfte, was prinzipiell ein bedeutsamer Hinweis darauf ist, dass diese Merkmale der Lehrkräfte zur Entwicklung des Wissens über Lehr-Lernmethoden und -konzepte beitragen könnten - inwiefern es sich hierbei um kausale Effekte handelt, kann mit den vorliegenden Querschnittsdaten nicht beantwortet werden. Denn für den Bereich der EB/WB gibt es derzeit keine Studien, die im Rahmen der derzeit eben nur angenommenen, aber nicht belegten Wirkungskette zwischen dem Wissen der Lehrkräfte und z.B. 
dem Lernerfolg der Teilnehmenden eine Verbindung herstellen können. Neben der begrenzten Stichprobengröße, die sich auch auf die Teststärke auswirkt, sind die Ergebnisse zudem unter dem Vorbehalt zu sehen, dass die Erfassung des Wissens über Lehr-Lernmethoden und -konzepte über die bereits vorgenommenen Validierungsschritte hinaus selbst noch weiter validiert werden muss.

Dennoch können die berichteten Befunde und methodischen Herausforderungen wichtige Anregungen geben für zukünftige und bestenfalls längsschnittliche Untersuchungen zur Frage nach der Entwicklung (von Aspekten) der professionellen Kompetenz von Lehrkräften.

\section{Anhang}

Tab. 4 und Abb. 3.

Tab. 4 Ergebnisse multipler latenter Regressionsanalysen (standardisierte Regressionsgewichte (STDY))

\begin{tabular}{|c|c|c|c|c|c|c|c|}
\hline & & $\mathrm{g}$ & A & B & $\mathrm{C}$ & $\mathrm{D}$ & $\mathrm{E}$ \\
\hline Alter & $\begin{array}{l}\beta \\
S E\end{array}$ & $\begin{array}{l}-0,012 \\
(0,053)\end{array}$ & $\begin{array}{l}-0,024 \\
(0,163)\end{array}$ & $\begin{array}{l}-0,024 \\
(0,173)\end{array}$ & $\begin{array}{l}-0,001 \\
(0,275)\end{array}$ & $\begin{array}{l}-0,043 \\
(0,219)\end{array}$ & $\begin{array}{l}0,004 \\
(0,294)\end{array}$ \\
\hline Geschlecht & $\begin{array}{l}\beta \\
S E\end{array}$ & $\begin{array}{l}-0,135 \\
(0,130)\end{array}$ & $\begin{array}{l}0,131 \\
(0,221)\end{array}$ & $\begin{array}{l}-0,163 \\
(0,298)\end{array}$ & $\begin{array}{l}0,064 \\
(0,149)\end{array}$ & $\begin{array}{l}0,068 \\
(0,290)\end{array}$ & $\begin{array}{l}-0,098 \\
(0,311)\end{array}$ \\
\hline $\begin{array}{l}\text { Tätigkeits- } \\
\text { schwerpunkt } \\
\text { EB oder WB }\end{array}$ & $\begin{array}{l}\beta \\
S E\end{array}$ & $\begin{array}{l}0,239 \\
(0,133)\end{array}$ & $\begin{array}{l}0,117 \\
(0,212)\end{array}$ & $\begin{array}{l}0,134 \\
(0,318)\end{array}$ & $\begin{array}{l}0,121 \\
(0,317)\end{array}$ & $\begin{array}{l}0,034 \\
(0,224)\end{array}$ & $\begin{array}{l}0,002 \\
(0,170)\end{array}$ \\
\hline $\begin{array}{l}\text { Aktueller Lehr- } \\
\text { umfang in Wo- } \\
\text { chenstunden }\end{array}$ & $\begin{array}{l}\beta \\
S E\end{array}$ & $\begin{array}{l}0,015 \\
(0,155)\end{array}$ & $\begin{array}{l}0,017 \\
(0,397)\end{array}$ & $\begin{array}{l}0,163 \\
(0,354)\end{array}$ & $\begin{array}{l}-0,234 \\
(0,150)\end{array}$ & $\begin{array}{l}0,033 \\
(0,302)\end{array}$ & $\begin{array}{l}0,026 \\
(0,251)\end{array}$ \\
\hline $\begin{array}{l}\text { Erziehungs- und } \\
\text { bildungswiss. } \\
\text { Studium }\end{array}$ & $\begin{array}{l}\beta \\
S E\end{array}$ & $\begin{array}{l}-0,086 \\
(0,153)\end{array}$ & $\begin{array}{l}-0,114 \\
(0,232)\end{array}$ & $\begin{array}{l}0,080 \\
(0,406)\end{array}$ & $\begin{array}{l}0,128 \\
(0,153)\end{array}$ & $\begin{array}{l}0,092 \\
(0,409)\end{array}$ & $\begin{array}{l}-0,016 \\
(0,172)\end{array}$ \\
\hline $\begin{array}{l}\text { Lehrerfahrung in } \\
\text { Jahren }\end{array}$ & $\begin{array}{l}\beta \\
S E\end{array}$ & $\begin{array}{l}-0,075 \\
(0,204)\end{array}$ & $\begin{array}{l}0,223 \\
(0,269)\end{array}$ & $\begin{array}{l}-0,182 \\
(0,400)\end{array}$ & $\begin{array}{l}0,087 \\
(0,184)\end{array}$ & $\begin{array}{l}-0,226 \\
(0,531)\end{array}$ & $\begin{array}{l}0,022 \\
(0,502)\end{array}$ \\
\hline $\begin{array}{l}\text { Stunden Fort- } \\
\text { und Weiterbil- } \\
\text { dung }\end{array}$ & $\begin{array}{l}\beta \\
S E\end{array}$ & $\begin{array}{l}0,232 \\
(0,329)\end{array}$ & $\begin{array}{l}-0,279 \\
(0,203)\end{array}$ & $\begin{array}{l}0,181 \\
(0,513)\end{array}$ & $\begin{array}{l}0,091 \\
(0,547)\end{array}$ & $\begin{array}{l}0,186 \\
(0,428)\end{array}$ & $\begin{array}{l}0,042 \\
(0,906)\end{array}$ \\
\hline $\begin{array}{l}\text { Interaktion Lehr- } \\
\text { erfahrung und } \\
\text { Fort- und Weiter- } \\
\text { bildung }\end{array}$ & $\begin{array}{l}\beta \\
S E\end{array}$ & $\begin{array}{c}-0,265 \\
(0,318)\end{array}$ & $\begin{array}{l}0,048 \\
(0,260)\end{array}$ & $\begin{array}{c}-0,036 \\
(0,581)\end{array}$ & $\begin{array}{c}-0,088 \\
(0,532)\end{array}$ & $\begin{array}{c}-0,102 \\
(0,657)\end{array}$ & $\begin{array}{c}-0,029 \\
(0,557)\end{array}$ \\
\hline
\end{tabular}




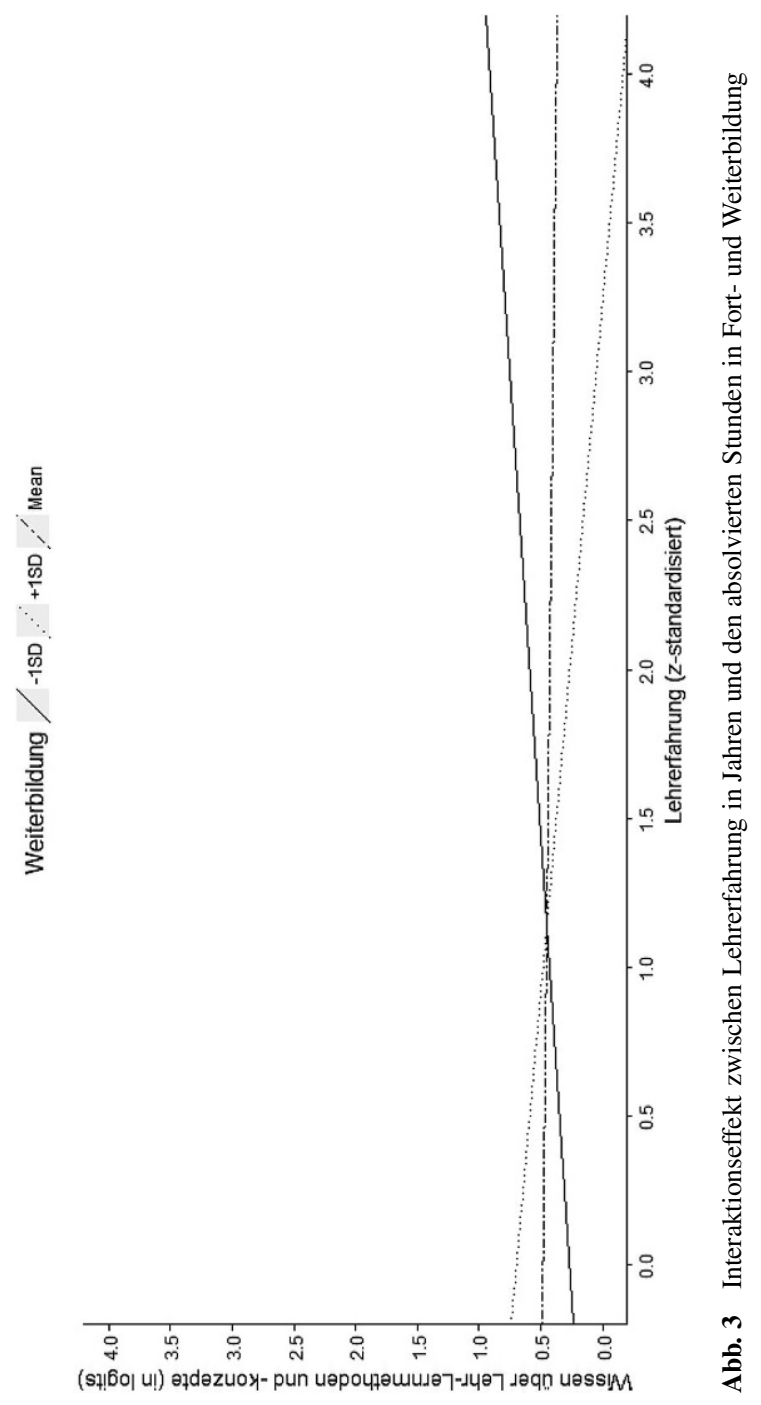


Open Access Dieser Artikel wird unter der Creative Commons Namensnennung 4.0 International Lizenz (http://creativecommons.org/licenses/by/4.0/deed.de) veröffentlicht, welche die Nutzung, Vervielfältigung, Bearbeitung, Verbreitung und Wiedergabe in jeglichem Medium und Format erlaubt, sofern Sie den/die ursprünglichen Autor(en) und die Quelle ordnungsgemäß nennen, einen Link zur Creative Commons Lizenz beifügen und angeben, ob Änderungen vorgenommen wurden.

\section{Literatur}

Aebli, H. (2011). Zwölf Grundformen des Lehrens. Eine allgemeine Didaktik auf psychologischer Grundlage. Medien und Inhalte didaktischer Kommunikation, der Lernzyklus (14. Aufl.). Stuttgart: KlettCotta.

Arnold, R., Krämer-Stürzl, A., \& Siebert, H. (2005). Dozentenleitfaden. Planung und Unterrichtsvorbereitung in Fortbildung und Erwachsenenbildung. Berlin: Cornelsen.

Autorengruppe Bildungsberichterstattung (2016). Bildung in Deutschland 2016. Ein indikatorengestützter Bericht mit einer Analyse zu Bildung und Migration (1. Aufl.). Bielefeld: wbv.

Autorengruppe wb-personalmonitor (2016). Personal in der Weiterbildung: Beschäftigungsverhältnisse und Tätigkeiten - wb-personalmonitor. Bielefeld: wbv.

Avramovska, M., Czerwinski, T., \& Lattke, S. (2015). Curriculum globALE. Global curriculum for Adult Learning and Education (2. Aufl.). Bonn: DVV International, DIE.

Baumert, J., \& Kunter, M. (2011). Das Kompetenzmodell von COACTIV. In M. Kunter, J. Baumert, W. Blum, U. Klusmann, S. Krauss \& M. Neubrand (Hrsg.), Professionelle Kompetenz von Lehrkräften. Ergebnisse des Forschungsprogramms COACTIV (S. 29-54). Münster: Waxmann.

Chen, F.F., West, S.G., \& Sousa, K.H. (2006). A comparison of bifactor and second-order models of quality of life. Multivariate Behavioral Research, 41(2), 189-225.

Collins, J.B., \& Pratt, D.D. (2011). The teaching perspectives inventory at 10 years and 100,000 respondents. Adult Education Quarterly, 61(4), 358-375.

Digel, S. (2013). Von der Diagnose zum Handeln: Befunde aus einer Interventionsstudie zum Lernen mit Videofällen. In S. Digel \& J. Schrader (Hrsg.), Diagnostizieren und Handeln von Lehrkräften. Lernen aus Videofällen in Hochschule und Erwachsenenbildung (S. 25-61). Bielefeld: wbv.

Dobischat, R., Elias, A., \& Rosendahl, A. (Hrsg.). (2018). Das Personal in der Weiterbildung. Im Spannungsfeld von Professionsanspruch und Beschäftigungsrealität. Wiesbaden: Springer.

European Commission (2015). Strengthening teaching in Europe. New evidence from teachers compiled by Eurydice and CRELL. http://ec.europa.eu/education/library/policy/teaching-professionpractices_en.pdf. Zugegriffen: 22. Mai 2018.

Fuchs, S., Kollmansberger, M., Schwickerath, A., Barz, H., v. Hippel, A., \& Tippelt, R. (2009). Fortbildungsinteressen und -bedarfe. In A. v. Hippel \& R. Tippelt (Hrsg.), Fortbildung der Weiterbildner/ innen. Eine Analyse der Interessen und Bedarfe aus verschiedenen Perspektiven (S. 143-199). Weinheim und Basel: Beltz.

Goeze, A. (2016). Professionalitätsentwicklung von Lehrkräften durch videofallbasiertes Lernen - Voraussetzungen, Prozesse, Wirkungen. Bielefeld: wbv.

Goeze, A., Hetfleisch, P., \& Schrader, J. (2013). Wirkungen des Lernens mit Videofällen bei Lehrkräften. Zeitschrift für Erziehungswissenschaft, 16(1), 79-113.

Großschedl, J., Harms, U., Kleickmann, T., \& Glowinski, I. (2015). Preservice biology teachers' professional knowledge. Structure and learning opportunities. Journal of Science Teacher Education, 26(3), 291-318.

Gruber, H., Mandl, H., \& Renkl, A. (2000). Was lernen wir in Schule und Hochschule: Träges Wissen? In H. Mandl \& J. Gerstenmaier (Hrsg.), Die Kluft zwischen Wissen und Handeln: Empirische und theoretische Lösungsansätze (S. 139-156). Göttingen: Hogrefe.

Guerriero, S. (Hrsg.). (2017). Pedagogical knowledge and the changing nature of the teaching profession. Paris: OECD Publishing.

Hartz, S. (2011). Qualität in Organisationen der Weiterbildung. Eine Studie zur Akzeptanz und Wirkung von $L Q W$ (1. Aufl.). Wiesbaden: VS.

Herrle, M., Dinkelaker, J., Nolda, S., \& Kade, J. (2014). Kursforschung und Videographie. In J. Kade, S. Nolda, J. Dinkelaker \& M. Herrle (Hrsg.), Videographische Kursforschung. Empirie des Lehrens und Lernens Erwachsener (S. 13-40). Stuttgart: Kohlhammer.

v. Hippel, A., \& Tippelt, R. (Hrsg.). (2009). Fortbildung der Weiterbildner/innen. Eine Analyse der Interessen und Bedarfe aus verschiedenen Perspektiven. Weinheim und Basel: Beltz. 
Hof, C. (2010). Wissen als Thema der Erwachsenenbildung. In B. Dewe (Hrsg.), Professionswissen und erwachsenenpädagogisches Handeln. Dokumentation der Jahrestagung 2001 der Sektion Erwachsenenbildung der Deutschen Gesellschaft für Erziehungswissenschaft (S. 9-17). Bielefeld: Bertelsmann.

Hoffmann, N. (2011). Ratgeber-Didaktik in Didaktik-Ratgebern. Portrait eines Selbstlernmediums aus erwachsenenpädagogischer Perspektive. Der pädagogische Blick, 19(1), 4-12.

Hohmann, R. (2010). Fortbildungsbedarf von Lehrkräften aus Sicht der KBE. In J. Schrader, R. Hohmann \& S. Hartz (Hrsg.), Mediengestützte Fallarbeit. Konzepte, Erfahrungen und Befunde zur Kompetenzentwicklung von Erwachsenenbildnern (S. 9-24). Bielefeld: wbv.

Kelava, A., \& Schermelleh-Engel, K. (2012). Latent-State-Trait-Theorie (LST). In H. Moosbrugger \& A. Kelava (Hrsg.), Testtheorie und Fragebogenkonstruktion (2. Aufl. S. 364-381). Heidelberg: Springer.

König, J., \& Blömeke, S. (2012). Future teachers' general pedagogical knowledge from a comparative perspective: Does school experience matter? ZDM Mathematics Education, 44(3), 341-354.

Kraft, S., Seitter, W., \& Kollewe, L. M. (2009). Professionalitätsentwicklung des Weiterbildungspersonals. Bielefeld: wbv.

Lattke, S., \& Jütte, W. (Hrsg.). (2014). Professionalisation of adult educators: International and comparative perspectives. Frankfurt a.M.: Peter Lang.

Lencer, S., \& Strauch, A. (2016). Das GRETA-Kompetenzmodell für Lehrende in der Erwachsenenund Weiterbildung. www.die-bonn.de/doks/2016-erwachsenenbildung-02.pdf. Zugegriffen: 22. Mai 2018.

Lenske, G., Thillmann, H., Wirth, J., Dicke, T., \& Leutner, D. (2015). Pädagogisch-psychologisches Professionswissen von Lehrkräften. Evaluation des ProwiN-Tests. Zeitschrift für Erziehungswissenschaft, 18(2), 225-245.

Lüdtke, O., Robitzsch, A., Trautwein, U., \& Köller, O. (2007). Umgang mit fehlenden Werten in der psychologischen Forschung. Psychologische Rundschau, 58(2), 103-117.

Marx, C., Goeze, A., \& Schrader, J. (2014a). Adult education teachers' pedagogical psychological knowledge: Elements and test development. In S. Lattke \& W. Jütte (Hrsg.), Professionalisation of adult educators: International and comparative perspectives (S. 162-182). Frankfurt a.M.: Peter Lang.

Marx, C., Goeze, A., \& Schrader, J. (2014b). Pädagogisch-psychologisches Wissen zur Gestaltung von Lehr-Lernsituationen: (Wie) Unterscheidet es sich in Erwachsenenbildung/Weiterbildung und Schule? Hessische Blätter für Volksbildung, 64(3), 238-251.

Marx, C., Goeze, A., Voss, T., Hoehne, V., Klotz, V. K., \& Schrader, J. (2017). Pädagogisch-psychologisches Wissen von Lehrkräften aus Schule und Erwachsenenbildung. Entwicklung und Erprobung eines Testinstruments. Zeitschrift für Erziehungswissenschaft, 20(1), 165-200.

Marx, C., Goeze, A., Voss, T., Deutscher, V. K., Kelava, A., \& Schrader, J. (2018). Das Wissen über LehrLernmethoden von Lehrkräften der Erwachsenen- und Weiterbildung - Wie lässt sich die Dimensionalität valide abbilden? eingereicht.

Muthén, L.K., \& Muthén, B.O. (2015). Mplus user's guide (7. Aufl.). Los Angeles, CA: Muthén und Muthén.

Neumann, M., Schnyder, I., Trautwein, U., Niggli, A., Lüdtke, O., \& Cathomas, R. (2007). Schulformen als differenzielle Lernmilieus. Zeitschrift für Erziehungswissenschaft, 10(3), 399-420.

Nittel, D., Schütz, J., Fuchs, S., \& Tippelt, R. (2011). Die Orientierungskraft des Lebenslangen Lernens bei Weiterbildnern und Grundschullehrern. Erste Befunde aus dem Forschungsprojekt PAELL. In W. Helsper \& R. Tippelt (Hrsg.), Pädagogische Professionalität (Bd. 57, S. 167-183). Weinheim: Beltz. Zeitschrift für Pädagogik. Beiheft.

Pachner, A. (2013). Selbstreflexionskompetenz. Voraussetzung für Lernen und Veränderung in der Erwachsenenbildung? Magazin erwachsenenbildung.at, 20, 1-7. https://www.pedocs.de/volltexte/ 2013/8410/pdf/Erwachsenenbildung_20_2013_Pachner_Selbstreflexionskompetenz.pdf

Rivkin, S. G., Hanushek, E. A., \& Kain, J.F. (2005). Teachers, schools, and academic achievement. Econometrica, 73(2), 417-458.

Rohs, M., Rott, K.J., Schmidt-Hertha, B., \& Bolten, R. (2017). Medienpädagogische Kompetenzen von ErwachsenenbildnerInnen. Magazin erwachsenenbildung.at, 30, 1-14.

v. Rosenbladt, B., \& Lehmann, R. (2013). Grade der Schriftbeherrschung und subjektiver Lernerfolg bei Teilnehmenden an Alphabetisierungskursen. Zeitschrift für Erziehungswissenschaft, 16(1), 55-77.

Schläfli, A., \& Sgier, I. (2008). Porträt Weiterbildung Schweiz. Bielefeld: wbv.

Schöb, S., Rohs, M., Biel, C., \& Scholze, T. (2016). Professionalisierung von Lehrhandeln in einer digital geprägten Lernkultur - Bedarf und Ansatzpunkte der Entwicklung einer Online-Lernumgebung. In O. Dörner, C. Iller, H. Pätzold \& S. Robak (Hrsg.), Differente Lernkulturen - regional, national, transnational (S. 171-183). Opladen: Barbara Budrich. 
Schrader, J., Anders, Y., \& Richter, D. Fortbildung des pädagogischen Personals in der frühen Bildung, der Schule und der Erwachsenen- und Weiterbildung (2018). In O. Köller, M. Hasselhorn, F. W. Hesse, K. Maaz, J. Schrader \& H. Solga, et al. (Hrsg.), Das Bildungswesen in Deutschland. Bestand und Potenziale. Bad Heilbrunn: UTB. im Druck.

Schrader, J., \& Goeze, A. (2018). Professionelle Kompetenzen von Lehrkräften in der Erwachsenen- und Weiterbildung - Ein Rahmenmodell für Forschung, Rekrutierung und Fortbildung. in Vorbereitung

Schütz, J., \& Nittel, D. (2012). Von der Heterogenität zur Vielfalt. Akademische Professionalisierung im Blick einer komparativen pädagogischen Berufsgruppenforschung. In R. Egetenmeyer \& I. Schüßler (Hrsg.), Akademische Professionalisierung in der Erwachsenenbildung/Weiterbildung. Grundlagen der Berufs- und Erwachsenenbildung, (Bd. 70, S. 229-244). Baltmannsweiler: Schneider.

Shulman, L.S. (1986). Those who understand: Knowledge growth in teaching. Educational Researcher, $15,4-14$.

Skott, J. (2015). The promises, problems, and prospects of research on teachers' beliefs. In H. Fives \& M. G. Gill (Hrsg.), International handbook of research on teachers' beliefs. Educational psychology handbook series. (S. 13-30). New York: Routledge.

Sorge, S., Kröger, J., Petersen, S., \& Neumann, K. (2017). Structure and development of pre-service physics teachers' professional knowledge. International Journal of Science Education. https://doi.org/ 10.1080/09500693.2017.1346326

Stanik, T. (2016). Mikrodidaktische Planungen von Lehrenden in der Erwachsenenbildung. Zeitschrift für Weiterbildungsforschung - Report, 39(3), 317-330.

Steiner, P. (2010). Erfahrungen und Zugänge zu Beratung und Kompetenzanerkennung für ErwachsenenbildnerInnen. Magazin erwachsenenbildung.at, 14, 1-6. https://erwachsenenbildung.at/magazin/1009/meb10-9_14_steiner.pdf

Terhart, E. (2012). Wie wirkt Lehrerbildung? Forschungsprobleme und Gestaltungsfragen. Zeitschrift für Bildungsforschung, 2(1), 3-21.

Terhart, E. (2013). Neuansätze in der Allgemeinen Didaktik: Ein Kommentar. Jahrbuch für Allgemeine Didaktik, 3, 219-230.

Tietgens, H. (1967). Schule und Erwachsenenbildung. Braunschweig: Georg Westermann.

Vinepac-Project (2008). Handbook for the use of Validpack for the validation of psycho-pedagogical adult educators' competences. Timisoara: Editura Mirton.

Voss, T., Kunter, M., \& Baumert, J. (2011). Assessing teacher candidates' general pedagogical/psychological knowledge: Test construction and validation. Journal of Educational Psychology, 103(4), 952-969.

Voss, T., Kunina-Habenicht, O., Hoehne, V., \& Kunter, M. (2015). Stichwort Pädagogisches Wissen von Lehrkräften: Empirische Zugänge und Befunde. Zeitschrift für Erziehungswissenschaft, 18(2), $187-223$.

Voss, T., Goeze, A., Marx, C., Hoehne, V., Klotz, V.K., \& Schrader, J. (2017). Using digital media to assess and promote school and adult education teacher competence. In J. Buder \& F. W. Hesse (Hrsg.), Informational Environments (S. 125-148). Cham: Springer International Publishing AG.

Wainer, H., \& Kiely, G.L. (1987). Item clusters and computerized adaptive testing: A case for testlets. Journal of Educational Measurement, 24(3), 185-201.

Wang, W.-C., \& Wilson, M. (2005). The Rasch testlet model. Applied Psychological Measurement, 29(2), $126-149$.

Weinert, F. E. (2001). Concept of competence: A conceptual clarification. In D. S. Rychen \& L. H. Salganik (Hrsg.), Defining and selecting key competencies (S. 45-65). Seattle: Hogrefe und Huber.

Wißhak, S. (2017). Qualifizierung und professionelles Wissen von Lehrpersonen in der berufsbezogenen Weiterbildung. http://nbn-resolving.de/urn:nbn:de:bsz:352-2--qjhzazjd0xb83. Zugegriffen: 22. Mai 2018.

Wißhak, S., \& Hochholdinger, S. (2016). Analyse der Inhalte erziehungswissenschaftlicher Studiengänge im Hinblick auf eine spätere Tätigkeit in der berufsbezogenen Weiterbildung. Zeitschrift für Weiterbildungsforschung - Report, 39(1), 97-115.

Ziep, K.-D. (1990). Der Dozent in der Weiterbildung. Professionalisierung und Handlungskompetenzen. Weinheim: Deutscher Studien Verlag. 